\title{
Association of eating behavior with nutritional status and body composition in primary school-aged children
}

\begin{abstract}
Problematic eating behaviors during childhood may lead to positive energy balance and obesity. Therefore, this study aims to investigate the association of eating behaviors with nutritional status and body composition in Malaysian children aged 7 to 12 years. A total of 1782 primary schoolchildren were randomly recruited from 6 regions in Malaysia. The multidimensional Children's Eating Behaviour Questionnaire (CEBQ) was reported by parents to determine the 8 different dimensions of eating styles among children. Body mass index (BMI), BMI-for-age Z-score, waist circumference, and body fat percentage were assessed. Linear regression analyses revealed that both food responsiveness and desire to drink subscales were positively associated with a child's body adiposity, whereas satiety responsiveness, slowness in eating, and emotional undereating subscales were negatively associated with adiposity (all $\mathrm{P}<.05$ ). A multidimensional eating style approach based on the CEBQ is needed to promote healthy eating behaviors in order to prevent excessive weight gain and obesity problems among Malaysian children.
\end{abstract}

Keyword: Adiposity; Anthropometry; Body composition; Body mass index; Children; Eating behavior; Nutritional status; Waist circumference 